\title{
Couleur, travail et société, du Moyen Âge à nos jours
}

Paris/Lille, coédition Somogy éditions d'art, Archives départementales du Nord, Centre des archives du monde du travail, 2004, 233 p.

\section{Daisy Bonnard}

\section{(2) OpenEdition}

1 Journals

Édition électronique

URL : http://journals.openedition.org/dht/346

DOI : $10.4000 /$ dht.346

ISSN : 1775-4194

Éditeur :

Centre d'histoire des techniques et de l'environnement du Cnam (CDHTE-Cnam), Société des élèves du CDHTE-Cnam

Édition imprimée

Date de publication : 1 décembre 2009

Pagination : 221-222

ISBN : 978-2-9530779-4-0

ISSN : 0417-8726

\section{Référence électronique}

Daisy Bonnard, «Couleur, travail et société, du Moyen Âge à nos jours », Documents pour l'histoire des techniques [En ligne], 18| $2^{\mathrm{e}}$ semestre 2009, mis en ligne le 24 septembre 2010, consulté le 24 septembre 2020. URL : http://journals.openedition.org/dht/346; DOI : https://doi.org/10.4000/dht. 346

Ce document a été généré automatiquement le 24 septembre 2020.

(c) Tous droits réservés 


\section{Couleur, travail et société, du Moyen Âge à nos jours}

Paris/Lille, coédition Somogy éditions d'art, Archives départementales du Nord, Centre des archives du monde du travail, 2004, $233 \mathrm{p}$.

Daisy Bonnard

\section{RÉFÉRENCE}

Couleur, travail et société, du Moyen Âge à nos jours, Paris/Lille, coédition Somogy éditions d'art, Archives départementales du Nord, Centre des archives du monde du travail, $2004,233 \mathrm{p}$. 
1 Le Conseil Général du Nord, dans le cadre $\mathrm{du}$ programme «Lille 2004, capitale européenne de la culture », a organisé une exposition "Couleur, travail et société " en partenariat avec les Archives départementales et le Centre des archives du monde du travail. La réussite de cette exposition a été facilitée par le prêt d'œuvres et de documents non seulement de la part des musées, des bibliothèques ou de différents lieux d'archives mais également par des dépôts privés. De cette exposition est né un ouvrage, du même

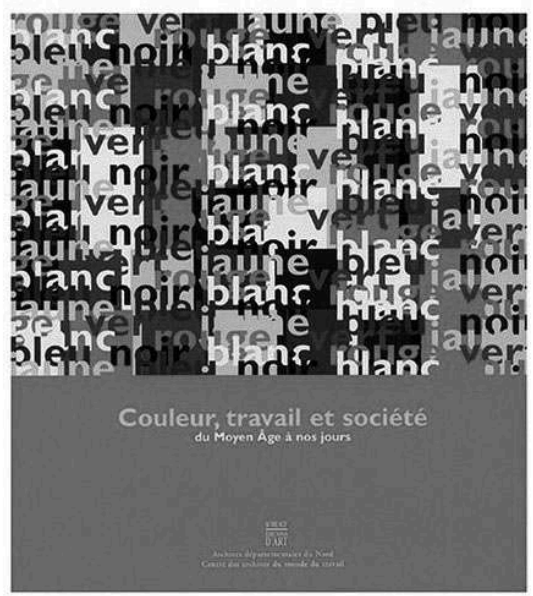
nom, évoquant les techniques et leurs conditions de production, les différents corps de métiers, le commerce de la couleur et la publicité.

2 Dans l'introduction, "Pour une histoire sociale des couleurs", Michel Pastoureau, historien réputé des couleurs ${ }^{1}$, campe la problématique de cet ouvrage collectif: l'histoire des couleurs ne peut se limiter au champ pictural ou artistique, elle est avant tout celle des teinturiers, des maîtres verriers, des tailleurs, des imprimeurs et autres artisans et corps de métiers qui ont plus à nous apprendre sur l'histoire de la couleur que les seuls artistes peintres.

3 Le livre, en deux parties, offre onze articles rédigés par les membres du comité scientifique de l'exposition, suivis d'un catalogue raisonné. Dans la préface et l'avantpropos, il est écrit que l'exposition (et ce livre qui en découle) a été une belle occasion pour mettre à jour des documents méconnus et que les archives ont occupé une place centrale. Si l'utilisation des illustrations et la reproduction de documents des Archives départementales et du Centre des archives du monde du travail est conséquente et magnifique, au fil de la lecture de ce recueil, on est en droit de regretter le choix de certains articles : il est bien dommage de ne pas avoir utilisé plus amplement ces fonds d'archives pour développer l'histoire industrielle de la région du Nord.

Si les sujets des divers articles ont déjà fait l'objet de plusieurs publications, comme celui d'André Dhainaut qui a trait aux travaux d'Isaac Newton, de Thomas Young ou de Johann Goethe sur la perception de la couleur, ou encore celui d'Alain Chrisment, très technique, sur la colorimétrie, dans laquelle les thèmes abordés auraient pu rejoindre le contexte régional, certaines contributions, soit par le langage simple et accessible de l'auteur, soit par une approche différente et originale, apportent un regard nouveau. C'est le cas de l'histoire que propose Bruno Vouters de la création des vitraux de l'École maternelle de Cateau-Cambrésis (ville natale d'Henri Matisse), née de la rencontre du peintre et d'un dominicain passionné d'art, Louis Rayssigier.

5 Didier Terrier, dans son article "du 'demi-blanc' au 'dernier blanc' ", nous emmène dans la France septentrionale du XVIII e siècle, avec le blanchiment des toiles de lin. Trempage du lin dans l'eau courante, séchage puis lessivage dans plusieurs bains, dont les bains de cendre, puis étendage des toiles dans les prés où elles sont régulièrement aspergées : le blanchiment est une tache laborieuse. Dans cette région où le tissage du lin occupe une place importante, la nécessité de développer les blanchisseries s'imposent afin de barrer l'envoi des toiles à blanchir en Flandre étrangère. Ainsi, 
l'activité va non seulement s'accroitre mais également s'améliorer et fera la fortune de bien des blanchisseurs du côté de Valenciennes.

6 Avec Corine Maitte, nous passons au rouge. Les recettes et secrets à partir de la garance, de la pourpre, du kermès ou des cochenilles de Pologne et d'Arménie teintent et colorent les textiles, jusqu'à la découverte du Nouveau Monde, dont la cochenille, du Mexique, va révolutionner les méthodes de teinture. Cependant, à l'heure où l'indienne est en vogue, chimistes et teinturiers européens sont à la recherche d'un secret de fabrication, celui d'un rouge magnifique sur coton : le « rouge turc ».

7 Gérard Gayot, récemment disparu, spécialiste de l'industrie textile, nous conduit à Sedan, réputée pour ses teintures au noir, et qu'il a étudiée de près tout au long de sa carrière. Si le drap de Sedan était déjà très estimé en Europe sous Colbert, sa réputation est inégalée à la mort de Louis XVIII lorsque les besoins pressants de draps noirs nécessitent la contribution des autres villes. La supériorité et la beauté du noir de Sedan sont incontestées.

8 Enfin, Amandine Delcourt dresse un historique des relations des industriels avec les arts à Roubaix. Les manufacturiers de la ville, conscients de la valeur patrimoniale de la création textile locale, fondent dès 1835, le premier musée industriel. En 1881, l'Ecole nationale des arts industriels ouvre ses portes. Après l'initiative du musée, les industriels fondent la Société artistique de Roubaix-Tourcoing et organisent un premier salon des beaux-arts dans le but de « développer le goût des arts, en favoriser les progrès et aider ainsi à l'éducation du public en lui procurant des distractions utiles et agréables ». Au fil des années, le salon prend de l'importance et l'on compte jusqu'à trois cent dix-huit exposants en 1896, jusqu'au milieu du XXe siècle, quand il perd de son prestige et laisse la place aux galeries d'art. En 2001, afin de renouer avec le passé artistique de Roubaix et ses salons, la ville inaugure dans l'ancienne piscine le nouveau musée d'Art et d'Industrie.

Suit un catalogue raisonné, conçu autour de six couleurs (jaune, bleu, noir, blanc, rouge, vert) Les auteurs ont réservé un chapitre par couleur, conçu sur une même trame: leur symbole, leur histoire et leur utilisation au fil des siècles ainsi que la caractéristique propre à chacune. L'étude tisse des analogies entre la couleur, le monde du travail et la société. Ainsi, la couleur jaune de même que l'or sont associés à la lumière et à la production d'images, et la couleur bleue à la teinture, le noir à l'habit de la vertu ou du travail, le blanc à la propreté et l'hygiène, le rouge à la lutte sociale et à la sécurité, le vert à l'architecture et l'entreprise. Chaque exemple exposé est illustré de publicités, de documents d'archives du Nord, nombreux, remarquables et inédits. Grâce au choix d'illustrations bien légendées, nous percevons toute l'importance de la couleur dans cette région industrielle. Ce catalogue raisonné, bien agencé, nous réconcilie avec l'ouvrage, dont la première partie nous semble moins convaincante. Au final, ce recueil collectif demeure avant tout un beau livre d'illustrations grand public, et sur ce point il atteint pleinement son objectif. 


\section{AUTEUR}

DAISY BONNARD

CDHTE-Cnam 